\title{
Prodotti della tradizione e contenuto di amine biogene alternative alla Low tyramine diet per la sostenibilità dei prodotti di nicchia e la salubrità del consumatore
}

\author{
Giovanna Suzzi, Rosanna Tofalo, Maria Schirone \\ Dipartimento di Scienze degli Alimenti, Università di Teramo, Italy
}

\begin{abstract}
Biogenic amines (BA) are present in a wide range of foods and mainly can be produced in high amounts by microorganisms through the activity of amino acid decarboxylases. Excessive consumption of foods with large concentrations of these compounds can induce adverse reactions such as nausea, headaches, rashes and changes in blood pressure. These problems are more severe in consumers with less efficient detoxification systems because of their genetic constitution or their medical treatments. The most common and powerful BA found in foods are histamine, tyramine and putrescine. Actually, there is no specific legislation regarding BA content in many fermented products, except for histamine; it is the only BA for which maximum levels in fish products have been set. So, it is generally assumed that these compounds should not be allowed to accumulate. Many factors such as bacterial density, synergistic effects between microorganisms, level of proteolysis (availability of substrate), $\mathrm{pH}$, salt, use of starter cultures, sanitization procedures adopted and conditions and time of ripening process are found to have limiting effects on the build-up of amines. Moreover, improved knowledge of the factors involved in the synthesis and accumulation of BA should lead to reduce in their incidence in foods.
\end{abstract}

\author{
Correspondence: Maria Schirone, Dipartimento di Scienze degli Alimenti, \\ Università di Teramo, via C.R. Lerici 1, 64023 Mosciano Sant'Angelo (TE), Italy. \\ Tel. +39.0861.266911 - Fax: +39.0861 .266940 . \\ Email: mschirone@unite.it \\ Key words: biogenic amines, fermented foods, tyramine. \\ Parole chiave: amine biogene, alimenti fermentati, tiramina. \\ Received for publication: 28 March 2011. \\ Accepted for publication: 9 May 2011. \\ Società rappresentata nell'ambito AISSA: Società Italiana di Microbiologia \\ Agraria, Alimentare e Ambientale - SI \\ (C) Copyright G. Suzzi et al., 2011 \\ Under no circumstances figures can be used without prior written consent \\ of the copyright owner. \\ Licensee PAGEPress, Italy \\ Italian Journal of Agronomy 2011; 6(s2):e8 \\ doi:10.4081/ija.2011.6.s2.e8
}

This work is licensed under a Creative Commons Attribution NonCommercial 3.0 License (CC BY-NC 3.0).

\section{Riassunto}

Le amine biogene (AB) sono presenti in molti alimenti fermentati e possono essere prodotte in grandi quantità dai microrganismi, attraverso specifiche attività aminoacido decarbossilasiche. L'eccessivo consumo di alimenti contenenti elevate concentrazioni di questi composti può indurre reazioni avverse come nausea, mal di testa, arrossamenti, ipotensione. Questi effetti possono diventare più severi in consumatori aventi un sistema di detossificazione meno efficiente per cause genetiche 0 perché trattati con farmaci inibitori. Le amine biogene più comuni negli alimenti sono istamina, tiramina e putrescina. Attualmente, non esiste un limite legislativo riguardo il contenuto di tali composti negli alimenti, fatta eccezione per l'istamina.

Molti sono i fattori che influenzano quantitativamente il contenuto di amine negli alimenti, come la densità microbica, gli effetti sinergici tra i microrganismi, il livello di proteolisi (disponibilità di substrato), il pH, il sale, l'aggiunta di colture starter, le procedure di sanitizzazione adottate e le condizioni e i tempi di maturazione. Lo studio di tali fattori può pertanto ridurre ed evitare la produzione e l'accumulo di tali composti.

Eseguendo una ricerca sul web con le parole Low tyramine diet, i motori di ricerca trovano più di 100.000 indicazioni. Ciò costituisce un rilevante segnale del problema tiramina nell'alimentazione e più in generale della presenza di amine biogene (AB) negli alimenti. Infatti, l'interesse per la tiramina e l'istamina nei prodotti alimentari è legato alle loro proprietà vasoattive che possono mettere a rischio la salute umana. Al momento, le persone sensibili sia all'istamina che alla tiramina sono incoraggiate a ridurre od eliminare l'ingestione di alimenti che possono contenere queste AB. Gli alimenti fermentati, ed in particolare i derivati della carne e del latte, costituiscono i prodotti con il maggior contenuto di AB (Tabella 1) (Ladero et al., 2010; Suzzi e Gardini, 2003).

\section{Effetti tossicologici}

Lintolleranza alla tiramina, ed anche all'istamina, è il risultato dell'accumulo di queste amine che non sono facilmente degradate dall'organismo. Infatti, se le AB introdotte con la dieta non sono eccessive, esse vengono metabolizzate attraverso un sistema di detossificazione basato su amino ossidasi epatiche ed intestinali. L'efficienza della detossificazione varia considerevolmente da individuo ad individuo e può essere condizionata e/o inibita da diversi fattori, quali il genotipo, l'eccessiva presenza di $\mathrm{AB}$ e l'assunzione di medicinali (Spano et al., 2010).

Un'eccessiva introduzione di $\mathrm{AB}$ con l'alimentazione provoca sin- 
tomi con effetti simili alle reazioni allergiche, come la diarrea, il mal di testa, le rinocongiuntiviti, l'asma, l'ipotensione, l'aritmia, l'orticaria, il prurito, l'arrossamento, etc. (Maintz e Novak, 2007).

\section{Valori soglia di tiramina}

La tiramina fu scoperta nel formaggio nel 1903 da Van Slyke e Hart, ma la prima relazione della sua presenza associata a reazioni ipertensive dopo ingestione di formaggio è stata riportata solo nel $1965 \mathrm{da}$ Blackwell e Mabbitt.

Nell'uomo sano, per innalzare la pressione sanguigna di almeno 30 $\mathrm{mmHg}$ è necessaria una somministrazione orale media di $500 \mathrm{mg} / \mathrm{kg}$ di tiramina (Ladero et al., 2010). Poiché le donne sembrano più sensibili a questo composto, la quantità richiesta per ottenere lo stesso effetto è più bassa. Comunque, una concentrazione pari a $125 \mathrm{mg} / \mathrm{kg}$ di tiramina è considerata tossica nelle persone normali tanto che è stato proposto un valore soglia di $100 \mathrm{mg} / \mathrm{kg}$ di tiramina e $30 \mathrm{mg} / \mathrm{kg}$ di feniletilamina (Ladero et al., 2010). Tuttavia, piuttosto che il valore di una singola amina, attualmente, sembra essere più utile indicare il valore massimo del contenuto totale di $\mathrm{AB}$ negli alimenti che dovrebbe essere compreso tra 750 e $900 \mathrm{mg} / \mathrm{kg}$ (ten Brink et al., 1990; Spanjer et al., 1991). Al momento l'unica $\mathrm{AB}$, per la quale è stato fissato un limite normativo, è l'istamina nei prodotti ittici. In particolare, il Regolamento CE n. 2073/2005 (relativo ai criteri microbiologici applicabili ai prodotti alimentari) definisce come valore limite 100 $200 \mathrm{mg} / \mathrm{kg}$ di istamina nei prodotti freschi della pesca che può arrivare fino a $400 \mathrm{mg} / \mathrm{kg}$ in quelli che hanno subito un trattamento di maturazione enzimatica in salamoia.

\section{La produzione di tiramina nel formaggi}

Le AB negli alimenti sono prodotte principalmente da microrganismi attraverso la decarbossilazione degli aminoacidi, ad eccezione delle poliamine fisiologiche (come spermina e spermidina). La quantità ed il tipo di amina prodotta dipende dall'alimento e dai microorganismi presenti (Ansorena et al., 2002; Ruiz-Capillas e JiménezColmenero, 2004). La capacità di decarbossilare gli aminoacidi è stata descritta in diversi generi, specie e ceppi di microrganismi (Ladero et al., 2010). I microrganismi con attività decarbossilasica nei formaggi possono essere batteri lattici (LAB), colture microbiche starter/non-starter od altri come enterococchi, micrococchi/stafilococchi e molti ceppi di Enterobacteriaceae (Halàsz et al., 1994; Galgano et al., 2001; Suzzi e Gardini, 2003). Tuttavia, è difficile trovare una correlazione diretta tra il numero di microrganismi ed il contenuto di $\mathrm{AB}$, poiché la capacità di produrre amine da parte dei diversi ceppi varia notevolmente (Innocente e D'Agostin, 2002; Schirone et al., 2011).

Istamina, tiramina, cadaverina, putrescina, triptamina e 2-feniletilamina sono state trovate in diversi tipi di formaggio (Silla Santos, 1996). Molti sono i fattori che contribuiscono alla presenza ed all'accumulo di $\mathrm{AB}$, come ad esempio la disponibilità di aminoacidi liberi, il pH, l'attività dell'acqua, il livello di salagione, la temperatura, la densità batterica e gli effetti sinergici tra i microrganismi (Suzzi e Gardini, 2003; Schirone et al., 2011).

È soprattutto nei formaggi tradizionali a latte crudo che le $\mathrm{AB}$ si accumulano a concentrazioni elevate (Martuscelli et al., 2005; Pintado et al., 2008; Schirone et al., 2011). In questi studi, come in numerosi altri, l'accumulo qualitativo e quantitativo di queste sostan-

Tabella 1. Contenuto di amine biogene in alimenti.

Table 1. Contents of biogenic amines in different foods.

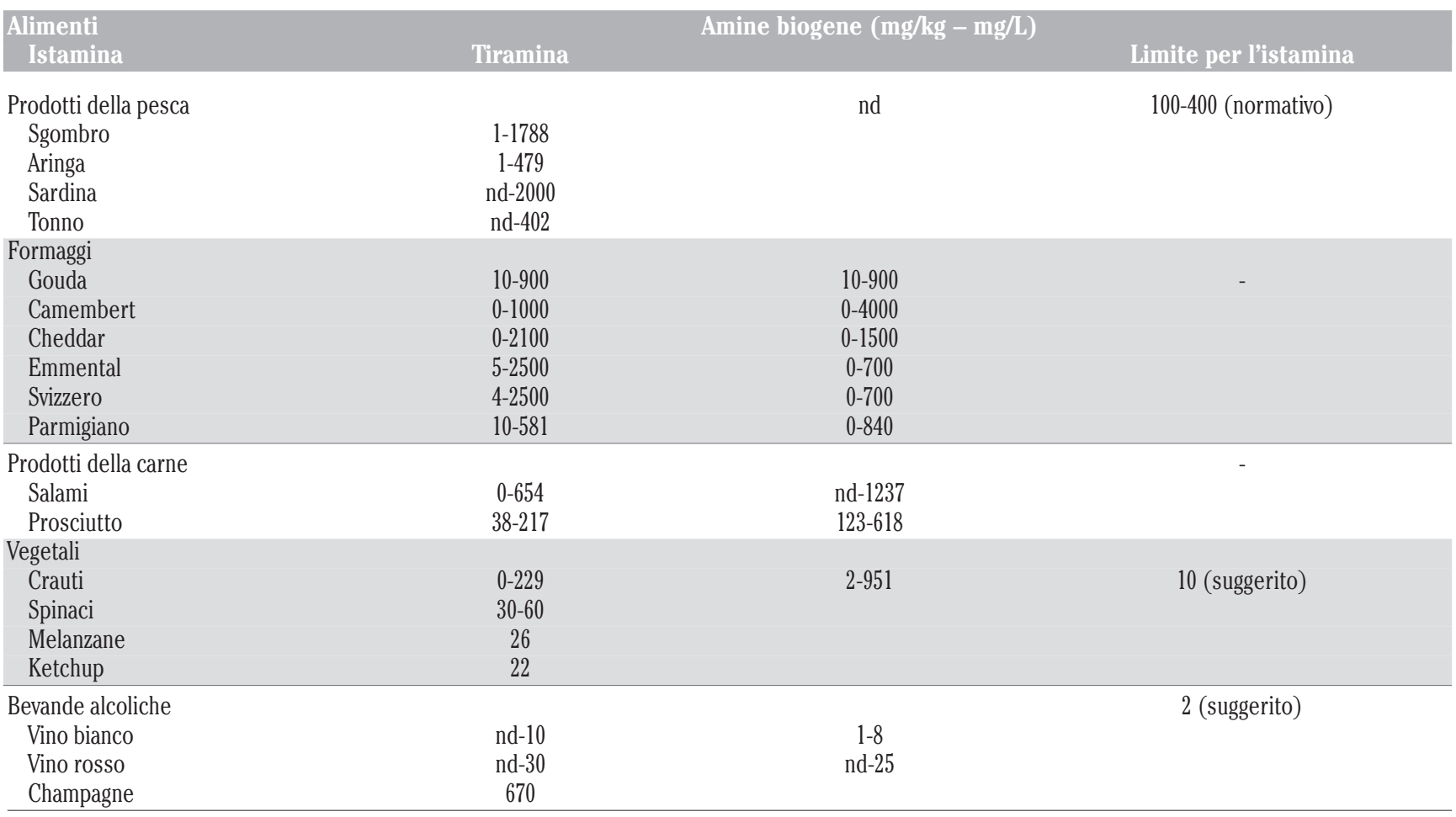

Modificato da Maintz e Novak (2007). 
ze è estremamente variabile e dipendente soprattutto da due fattori: i) la disponibilità di aminoacidi liberi prodotti man mano che la proteolisi procede a seconda del processo di caseificazione e ii) i microrganismi.

Nella Tabella 2 sono riportati i valori riguardanti il contenuto di $\mathrm{AB}$ in diversi formaggi italiani e non. Come si può osservare vi è una notevole diversità nei contenuti, ma è ben accertato che i formaggi freschi, così come pure lo yogurt, contengono poche amine o non ne contengono del tutto.

Dati analoghi possono essere osservati per quanto riguarda altri prodotti fermentati, come i salami, in cui, comunque, la fermentazione deve procedere a lungo ed è per questo più difficile impedire l'incremento dell'accumulo di AB.

\section{Fermentazione malolattica e produzione di amine biogene}

Nel settore enologico la produzione di AB è legata soprattutto al metabolismo dei $\mathrm{LAB}$, in quanto durante la fermentazione malolattica, processo che ha luogo nei vini rossi e talvolta, in quelli bianchi, vi è la formazione di $\mathrm{AB}$, in particolare di istamina (Lonvaud-Funel, 2001). Durante questo processo si ha la disacidificazione del vino

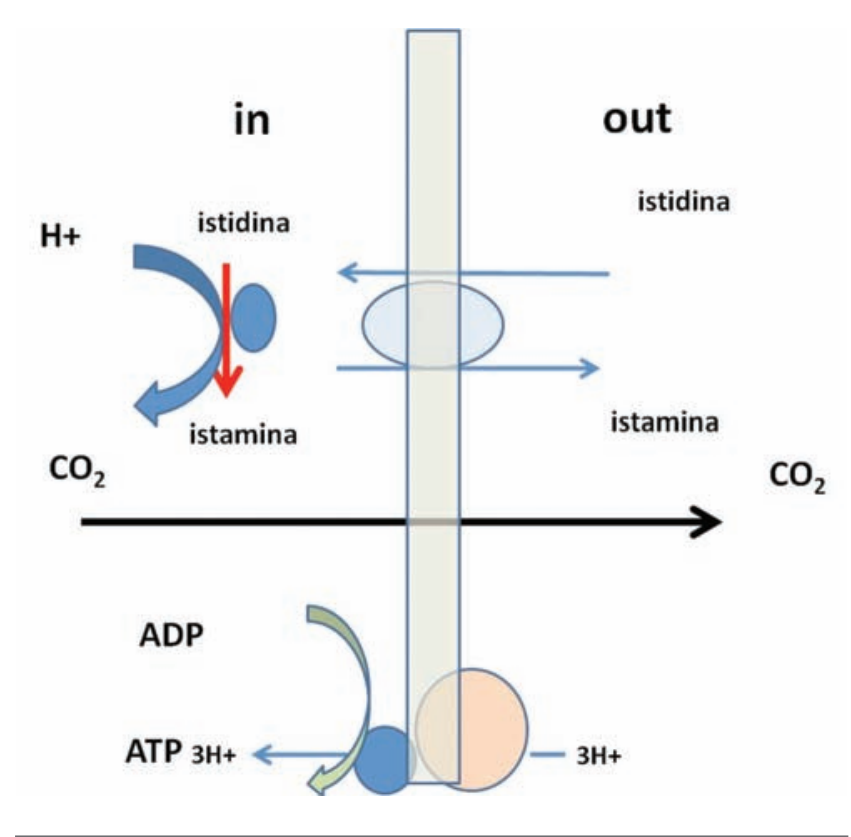

Figura 1. Decarbossilazione dell'istamina.

Figure 1. Metabolism of histamine.

Tabella 2. Amine biogene in prodotti lattiero-caseari.

Table 2. Biogenic amines in dairy products.

\begin{tabular}{|c|c|c|c|c|c|c|c|c|}
\hline \multicolumn{2}{|l|}{ Prodotti } & \multicolumn{5}{|c|}{ Amine biogene (mg/kg) } & \multirow{2}{*}{ Amine totali } & \multirow[t]{2}{*}{ Bibliografia } \\
\hline lattiero-caseari & Etilamina & Putrescina & Cadaverina & 2-feniletilamina & Tiramina & Istamina & & \\
\hline \multirow[t]{2}{*}{ Latte } & & 0,013 & & & & & & Novella-Rodríguez et al., 2004 \\
\hline & 4,87 & 17,92 & & 2,76 & & & & Lanciotti et al., 2007 \\
\hline Cagliata & - & 0,89 & 0,67 & & 2 & 0,98 & & Novella-Rodríguez et al., 2002 \\
\hline Siero & & 0,31 & 0,22 & & 0,65 & 0,28 & & Novella-Rodríguez et al., 2002 \\
\hline Pasta filata & & & & & & & $0,1-33,57$ & Giuffrida et al., 2006 \\
\hline Feta & - & 193 & 828 & 4,94 & 246 & 846 & - & Valsamaki et al., 2000 \\
\hline Pecorino Abruzzese & & & & $163-205$ & & $76-261$ & $600-1250$ & Martuscelli et al., 2005 \\
\hline Pecorino di Farindola & $12,9-601,3$ & $9,9-394$ & $26,8-276,1$ & $0-127,1$ & $52,3-1171,3$ & $0-21,8$ & 209-2393 & Schirone et al., 2011 \\
\hline Semicotto caprino & - & $85-115,7$ & $7,1-9,2$ & $18-22,2$ & $28,2-41,2$ & $17-23$ & 1313 & Galgano et al., 2001 \\
\hline Formaggio Portoghese & Terrincho & $31,0-153,3$ & $142,5-446,5$ & $48,6-239,6$ & $12,9-237,8$ & $0-283,1$ & $0-10,9$ & 428-922 Pintado et al., 2008 \\
\hline $\begin{array}{l}\text { Formaggio erborinato } \\
\text { da latte crudo } \\
\text { da latte pastorizzato }\end{array}$ & & $\begin{array}{l}875,8 \\
237,5\end{array}$ & $\begin{array}{c}756,7 \\
89,4\end{array}$ & 27,4 & $\begin{array}{c}1051 \\
526,6\end{array}$ & $\begin{array}{l}1041 \\
127\end{array}$ & - & Fernández et al., 2007 \\
\hline Formaggio A & & 35 & 4 & 12 & 72 & 10 & & Novella-Rodríguez et al., 2002 \\
\hline Formaggio B & & 78 & 7 & 17 & 98 & 10 & & Novella-Rodríguez et al., 2002 \\
\hline $\begin{array}{l}\text { Caciotta } \\
\text { a latte HPH } \\
\text { a latte termizzato } \\
\text { a latte crudo }\end{array}$ & & $\begin{array}{l}8,33 \\
8,30 \\
10,7 \\
\end{array}$ & $\begin{array}{c}10,1 \\
44,90 \\
24,9\end{array}$ & $\begin{array}{c}0 \\
3,87 \\
0\end{array}$ & $\begin{array}{l}28,0 \\
5,60 \\
8,97\end{array}$ & $\begin{array}{l}5,73 \\
7,30 \\
8,37\end{array}$ & & Lanciotti et al., 2007 \\
\hline $\begin{array}{l}\text { Pecorino } \\
\text { a latte HPH } \\
\text { a latte termizzato } \\
\text { a latte crudo }\end{array}$ & & $\begin{array}{l}14,80 \\
70,92 \\
29,3\end{array}$ & $\begin{array}{l}20,3 \\
257 \\
107\end{array}$ & $\begin{array}{l}19,5 \\
155 \\
63,3\end{array}$ & $\begin{array}{l}62,8 \\
350 \\
162\end{array}$ & $\begin{array}{l}3,35 \\
23,9 \\
6,32\end{array}$ & & Lanciotti et al., 2007 \\
\hline $\begin{array}{l}\text { Formaggio fresco } \\
\text { a latte crudo } \\
\text { a latte pastorizzato }\end{array}$ & & $\begin{array}{c}38,7 \\
\text { nd }\end{array}$ & $\begin{array}{c}96,3 \\
\text { nd }\end{array}$ & $\begin{array}{l}\text { nd } \\
\text { nd }\end{array}$ & $\begin{array}{c}233,3 \\
22\end{array}$ & $\begin{array}{c}110,8 \\
60,2\end{array}$ & & Fernández et al., 2007 \\
\hline $\begin{array}{l}\text { Formaggio stagionato } \\
\text { a latte crudo } \\
\text { a latte pastorizzato }\end{array}$ & & $\begin{array}{l}176,3 \\
175,3 \\
\end{array}$ & 328,4 & 40,7 & $\begin{array}{c}453,7 \\
301\end{array}$ & $\begin{array}{c}510,2 \\
65,4\end{array}$ & & Fernández et al., 2007 \\
\hline
\end{tabular}


attraverso la conversione dell'acido malico in acido lattico, ma possono verificarsi altri cambiamenti metabolici. Infatti, durante il loro sviluppo i LAB possono produrre AB; in particolare, Oenococcus oeni ne può produrre diverse in quantità rilevanti (Lonvaud-Funel, 2001; Guerrini et al., 2002). Anche se la quantità di $\mathrm{AB}$ nel vino è abbastanza bassa se confrontata con altri prodotti fermentati, lo studio di questi composti è particolarmente importante poiché l'etanolo può aumentare i suoi effetti tossici sul metabolismo umano inibendo le amino ossidasi. Istamina, tiramina e putrescina sono le principali $\mathrm{AB}$ presenti nei vini dove è avvenuta la fermentazione malolattica (Soufleros et al., 1998). Quindi, i vini contenenti amine biogene possono esaltare l'effetto di alimenti in cui sono presenti alti contenuti di $\mathrm{AB}$, come nei formaggi.

\section{Come possiamo controllare il contenuto di amine biogene nei prodotti fermentati?}

Sono numerosi i fattori che contribuiscono a modulare il tipo ed il contenuto di $\mathrm{AB}$ negli alimenti fermentati. In particolare, nei formaggi giocano un ruolo fondamentale le condizioni igieniche, la concentrazione di sale, il latte, crudo o pastorizzato, la temperatura e la durata della stagionatura, il contenuto in grasso, la carica batterica e l'aggiunta di colture starter. Tra i fattori chimico-fisici che possono influenzare l'accumulo di $\mathrm{AB}$ da parte dei microrganismi vi è il $\mathrm{pH}$ della matrice; infatti, è generalmente accettato che le vie di decarbossilazione sono attivate per aumentare la resistenza delle cellule a condizioni di sviluppo acide, per mantenere l'omeostasi del pH cellulare (Wolken et al., 2006). È stato individuato un locus genico coinvolto nella resistenza allo stress acido in Lactobacillus brevis, che contiene geni che formano due distinti operoni codificanti per vie ad attività aminoacido decarbossilasica (Lucas et al., 2007). Oltre ad essere coinvolte nella risposta allo stress acido, le vie di decarbossilazione degli aminoacidi possono essere responsabili di generazione di forza proton motrice (PMF). Generalmente, una PMF si genera per translocazione di protoni contro un gradiente attraverso la membrana cellulare. Meccanismi di questo tipo che coinvolgono la decarbossilazione di un aminoacido sono stati descritti per le coppie istidina/istamina e tirosina/tiramina (Pereira et al., 2009). La decarbossilasi converte l'aminoacido nella corrispondente amina e $\mathrm{CO}_{2}$ nel citoplasma, mentre la proteina trasportatore è responsabile dell'assorbimento dell'aminoacido dal mezzo e della secrezione della corrispondente $\mathrm{AB}$. Poiché viene consumato un protone nella reazione di decarbossilazione, essa contribuisce al mantenimento della omeostasi intracellulare del $\mathrm{pH}$ in ambiente acido. Inoltre, l'antiporter aminoacido/amina incrementa il potenziale di membrana (Figura 1) (Cotter e Hill, 2003).

Il numero di microorganismi presenti nella materia prima rimane, comunque, uno dei fattori critici; per cui il controllo delle $A B$ è spesso legato alla prevenzione dello sviluppo o dell'attività metabolica di quei microrganismi responsabili della loro formazione, mediante diverse processi tecnologici, come il riscaldamento, la salagione, l'aggiunta di spezie ed altro (Mah et al., 2009).

Un altro importante fattore suggerito, per prevenire l'accumulo di $A B$, è il controllo dei microrganismi produttori di queste sostanze attraverso l'uso di colture starter non producenti $\mathrm{AB}$ (Bover-Cid et al., 2000; Dapkevicius et al., 2000). Tuttavia, non tutti gli studi sono riusciti a dimostrare l'efficienza delle colture starter per ridurre la produzione di AB. Certamente un rapido ed intenso abbassamento del pH può spesso ridurre lo sviluppo dei microrganismi produttori all'inizio del processo fermentativo (Maijala et al., 1995). Tecnologie innovative utilizzate per ridurre il contenuto di $\mathrm{AB}$ nei prodotti caseari, quali l'uso dell'alta pressione pulsata (PSW) o l'alta pressione di omoge- neizzazione (HPH) hanno dato risultati promettenti per quanto riguarda la possibilità di inattivare microrganismi a temperatura ambiente 0 meno, anche per un ampio spettro di specie batteriche (Novella-Rodriguez et al., 2002; Lanciotti et al., 2007; Naila et al., 2010).

Per ciò che concerne il vino, un modo per prevenire il problema è stato proposto da Landete et al. (2007), in uno studio che suggerisce di ridurre al minimo quei processi che aumentano il contenuto di aminoacidi al mosto, come la macerazione delle bucce d'uva 0 il contatto con le fecce, inibendo i batteri lattici indigeni ed inoculando starter commerciali di $O$. oeni, non produttori di AB.

Molto importanti nello studio e nella ricerca sono i metodi utilizzati per la quantificazione delle AB (Önal, 2007), ma anche per l'individuazione rapida dei microrganismi produttori. Recentemente, sono state sviluppate metodiche basate sulla Real-Time PCR che hanno consentito di determinare e quantificare i microrganismi coinvolti nella produzione di alcune AB (Torriani et al., 2007; Gardini et al., 2008). In particolare, questo metodo è stato applicato per la valutazione dell'espressione dei geni codificanti per gli enzimi istidina decarbossilasi e tirosina decarbossilasi, $h d c A$ e $t d c A$ in salumi, formaggi e vino (Landete et al., 2007).

Studi molecolari riguardanti i microrganismi produttori di AB sono sempre più numerosi, rendendo più solide molte delle ipotesi fatte in passato, ma anche aprendo nuove strade per un controllo più mirato della produzione di $\mathrm{AB}$, che al momento rimane ancora parzialmente disatteso (Pereira et al., 2009; Coton et al., 2010).

\section{Bibliografia}

Ansorena D., Montel M.C., Rokka M., Talon R., Eerola S., Rizzo A., Raemaekers M., Demeyer D., 2002. Analysis of biogenic amines in Northern and Southern European sausages and role of flora in amine production. Meat Sci. 61:141-147.

Blackwell B., Mabbitt L., 1965. Tyramine in cheese related to hypertensive crisis after monoamine oxidase inhibition. Lancet 2:938.

Bover-Cid S., Izquierdo-Pulido M., Vidal-Carou M.C., 2000. Mixed starter cultures to control biogenic amine production in dry fermented sausages. J. Food Prot. 63:1556-1562.

Coton E., Mulder N., Coton M., Pochet S., Trip H., Lolkema J.S., 2010. Origin of the putrescine producing ability of the coagulase-negative bacterium Staphylococcus epidermidis 2015B. Appl. Environ. Microbiol. 76:5570-5576.

Cotter P.D., Hill C., 2003. Surviving the acid test: responses of Grampositive bacteria to low pH. Microbiol. Mol. Biol. Rev., 67: 429-453.

Dapkevicius M.L.N.E., Nout M.J.R., Rombouts F.M., Houben J.H., Wymenga W., 2000. Biogenic amine formation and degradation by potential fish slage starter microorganisms. Int. J. Food Microbiol. 57:107-114.

Fernández M., Linares D.M., Del Río B., Ladero V., Álvarez M.A., 2007. HPLC quantification of biogenic amines in cheeses: correlation with PCR-detection of tyramine-producing microorganism. J. Dairy Res. 74:276-282.

Galgano F., Suzzi G., Favati F., Caruso M.C., Martuscelli M., Gardini F., Salzano G., 2001. Biogenic amines during ripening in "Semicotto Caprino" cheese: role of enterococci. Int. J. Food Sci. Tech. $36: 153-160$.

Gardini F., Bover-Cid S., Tofalo R., Belletti N., Gatto V., Suzzi G., Torriani S., 2008. Modelling the aminogenic potential of Enterococcus faecalis EF37 in dry fermented sausages through chemical and molecular approaches. Appl. Environ. Microbiol. 74:2740-2750.

Giuffrida D., Ziino M., Verzera A., Condurso C., Romeo V., 2006. 
Biogenic amines in a typicali "Pasta filata" Italian cheese. Acta Aliment. 35:435-443.

Guerrini S., Mangani S., Granchi L., Vicenzini M., 2002. Biogenic amine production by Oenococcus oeni. Curr. Microbiol. 44:374378.

Halász A., Barath A., Simon-Sarkadi L., Holzapfel, W., 1994. Biogenic amines and their production by microorganisms in food. Trends Food Sci. Tech. 5:42-49.

Innocente N., D’Agostin P., 2002. Formation of biogenic amines in a typical semihard Italian heese. J. Food Prot. 65:1498-1501.

Ladero V., Calles-Enríquez M., Fernández M., Alvarez M.A., 2010. Toxicological effects of dietary biogenic amines. Curr. Nut. Food Sci. 6:145-156.

Lanciotti R., Patrignani F., Iucci L., Guerzoni M.E., Suzzi G., Belletti N., Gardini F., 2007. Effects of milk high pressure homogenization on biogenic amine accumulation during ripening of ovine and bovine Italian cheeses. Food Chem. 104:693-701.

Landete, J.M., De Las Rivas B., Marcobal A., Muñoz R., 2007. Molecular methods for the detection of biogenic amine producing bacteria on foods. Int. J. Food Microbiol. 117:258-269.

Lonvaud-Funel A., 2001. Biogenic amines in wine: role of lactic acid bacteria. FEMS Microbiol. Lett. 199:9-13.

Lucas P.M., Blancato V.S., Claisse 0., Magni C., Lolkema J.S., LonvaudFunel A., 2007. Agmatine deiminase pathway genes in Lactobacillus brevis are linked to the tyrosine decarboxylation operon in a putative acid resistance locus. Microbiol. 153:22212230.

Mah J.H., Hwang H.J., 2009. Inhibition of biogenic amine formation in a salted and fermented anchovy by Staphylococcus xylosus as a protective culture. Food Control 20:796-801.

Maijala R., Nurmi E., Fischer A., 1995. Influence of processing temperature on the formation of biogenic amines in dry sausages. Meat Sci. 39:9-22.

Maintz L., Novak N., 2007. Histamine and histamine intolerance. Am. J. Clin. Nutr. 85:1185-96.

Martuscelli M., Gardini F., Torriani S., Mastrocola D., Serio A., Chaves-Lopez C., Schirone M., Suzzi G., 2005. Production of biogenic amines during the ripening of Pecorino Abruzzese cheese. Int. Dairy J. 15:571-578.

Naila A., Flint S., Fletcher G., Bremer P., Meerdink G., 2010. Control of Biogenic amines in food - Exhisting and Emerging Approaches. J. Food Sci. 75:R139-R150.

Novella-Rodríguez S.N., Veciana-Nogues M.T., Roig-Sagues A.X., Trujillo-Mesa A.J., Vidal- Carou M.C., 2004. Evaluation of biogenic amines and microbial counts throughout the ripening of goat cheeses from pasteurized and raw milk. J. Dairy Res. 71:245-52.

Novella-Rodríguez S., Veciana-Nogués M.T., Saldo J., Vidal-Carou M.C., 2002. Effects of high hydrostatic pressure treatments on biogenic amine contents in goat cheeses during ripening. J. Agric. Food Chem. 50:7288-7292.
Önal A., 2007. A review: Current analytical methods for the determination of biogenic amines in foods. Food Chem. 103:1475-1486.

Pereira C.I., Matos D., San Romão M.V., Barreto Crespo M.T., 2009. Dual Role for the tyrosine decarboxylation pathway in Enterococcus faecium E17: response to an acid challenge and generation of a proton motive force. Appl. Environ. Microbiol. 75:345-352.

Pintado A.I.E., Pinho O., Ferreira I.M.P.L.V.O., Pintado M.M.E., Gomes A.M.P., Malcata F.X., 2008. Microbiological, biochemical and biogenic amine profiles of Terrincho cheese manufactured. Int. Dairy J. 18:631-640.

Regolamento CE No. 2073/2005 della Commissione del 15 novembre 2005 sui criteri microbiologici applicabili ai prodotti alimentari.

Ruiz-Capillas C., Jiménez-Colmenero F., 2004. Biogenic amines in meat and meat products. Crit. Rev. Food Sci. 44:489-499.

Schirone M., Tofalo R., Mazzone G., Corsetti A., Suzzi G., 2011. Biogenic amine content and microbiological profile of Pecorino di Farindola cheese. Food Microbiol. 28:128-136.

Silla Santos M.H., 1996. Biogenic amines: their importance in foods. Int. J. Food Microbiol. 29:213-231.

Soufleros E., Barrios M.L., Bertrand A., 1998. Correlation between the contento $\mathrm{f}$ biogenic amines and other wine compounds. Am. J. Enol. Vitic. 49:266-278.

Spanjer M.C., Van Roode B.A.S.W., 1991. Towards a regulatory limit for biogenic amines in fish, cheese and sauerkraut. De Ware(n)Chemicus 21:139-167.

Spano G., Russo P., Lonvaud-Funel A., Lucas P., Alexandre H., Grandvalet C., Coton E., Coton M., Barnavon L., Bach B., Rattray F., Bunte A., Magni C., Ladero V., Alvarez M., Fernández M., Lopez P., de Palencia P.F., Corbi A., Trip H., Lolkema J.S., 2010. Biogenic amines in fermented foods. Eur. J. Clin. Nutr. 64: S95-S100.

Suzzi G., Gardini F., 2003. Biogenic amines in dry fermented sausages: a review. I. J. Food Microbiol. 88:41-54.

ten Brink B., Damink C., Joosten H.M.L.J., Huis in’t Veld J.H.J., 1990. Occurrence and formation of biologically active amines in foods. Int. J. Food Microbiol. 11:73-84.

Torriani S., Gatto V., Sembeni S., Tofalo R., Suzzi G., Belletti N., Gardini F., Bover-Cid S., 2007. Rapid detection and quantification of tyrosine decarboxylase gene (tdc) and its expression in grampositive bacteria associated with fermented foods by PCR based methods J. Food Prot. 71:93-101.

Valsamaki K., Michaelidou A., Polychroniadou A., 2000. Biogenic amine production in Feta cheese. Food Chem. 71:259-266.

Van Slyke L.L., Hart E.B., 1903. The relation of carbon dioxide to proteolysis in the ripening of Cheddar cheese. NY State Agr. Expt. Sta. Bull. No. 231.

Wolken W.A.M., Lucas P.M., Lonvaud-Funel A., Lolkema J.S., 2006. The mechanism of the tyrosine transporter TyrP supports a proton motive tyrosine decarboxylation pathway in Lactobacillus brevis. J. Bacteriol. 188:2198-2206. 